\title{
Correction to: Application of the SureSelect target enrichment system for next-generation sequencing to obtain the complete genome sequence of bovine leukemia virus
}

\author{
Tung Duy Dao ${ }^{1,2} \cdot$ Vuong Nghia Bui $^{2} \cdot$ Tsutomu Omatsu $^{3} \cdot$ Yukie Katayama $^{3} \cdot$ Tetsuya Mizutani $^{3} \cdot$ Haruko Ogawa $^{1} \oplus$. \\ Kunitoshi Imai ${ }^{1}$
}

Published online: 22 August 2018

(c) Springer-Verlag GmbH Austria, part of Springer Nature 2018

\section{Correction to: Archives of Virology https://doi.org/10.1007/s00705-018-3957-9}

Unfortunately, Figure 1 was incorrectly published in the original publication and the correct version is updated here.

The original article can be found online at https://doi.org/10.1007/ s00705-018-3957-9.

Haruko Ogawa

hogawa@obihiro.ac.jp

1 Department of Veterinary Medicine, Obihiro University of Agriculture and Veterinary Medicine, 2-11 Inada, Obihiro, Hokkaido 080-8555, Japan

2 National Institute of Veterinary Research, 86 Truong Chinh, Dong Da, Hanoi, Vietnam

3 Research and Education Center for Prevention of Global Infectious Diseases of Animals, Tokyo University of Agriculture and Technology, Tokyo 183-8509, Japan 
Fig. 1 a. Maximum-likelihood phylogenetic tree constructed for the complete nucleotide sequences of four Vietnamese strains (indicated by $\bullet$ ), three Paraguayan strains (indicted by $\mathbf{\square}$ ), three Chinese strains (indicated by $\boldsymbol{\Delta}$ ), and reference strains obtained from the GenBank database. Genotypes G1-G10 are indicated on the right of the figure. The bar at the bottom of the figure denotes evolutionary distance. $\mathbf{b}$.

Maximum-likelihood phylogenetic tree for the complete BLV pol gene sequences of four Vietnamese strains (indicated by $\bullet$ ), three Paraguayan strains (indicated by $\mathbf{\square}$ ), three Chinese strains (indicated by $\boldsymbol{\Lambda}$ ), and reference strains obtained from the GenBank database. Genotypes G1-G10 are indicated on the right of the figure. The bar at the bottom of the figure denotes evolutionary distance. c. Maximum-likelihood phylogenetic tree for the complete BLV tax gene sequences of four Vietnamese strains (indicated by $\bullet$ ), three Paraguayan strains (indicated by $\boldsymbol{\square}$ ), three Chinese strains (indicated by $\boldsymbol{\Delta}$ ), and reference strains obtained from the GenBank database. Genotypes G1-G10 are indicated on the right of the figure. The bar at the bottom of the figure denotes evolutionary distance
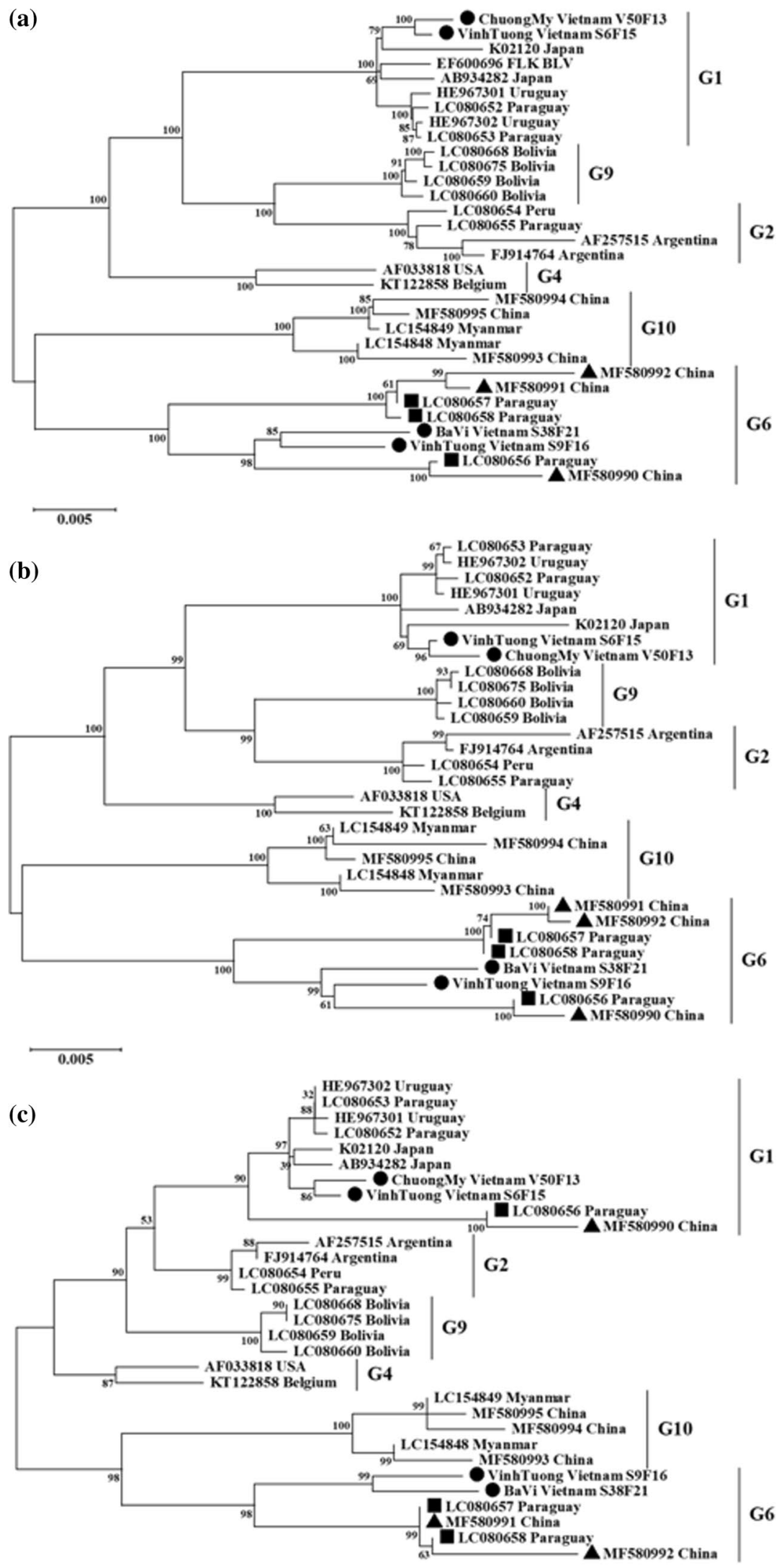